\title{
Local Allergic Rhinitis: Is There a Role for Systemic Allergy Immunotherapy?
}

Carmen Rondón, $M D, P h D^{1, *}$

Paloma Campo, MD, PhD ${ }^{1}$

Natalia López-Blanca, MD, $P h D^{2}$

María José Torres, MD, PhD ${ }^{1}$

Miguel Blanca, MD PhD ${ }^{1}$

\author{
Address \\ ${ }^{*}, 1$ Allergy Unit, Regional University Hospital of Malaga, IBIMA, UMA, \\ Malaga, Spain \\ Email: carmenrs61@gmail.com \\ ${ }^{2}$ Allergy Unit, Infanta Leonor Hospital, Madrid, Spain
}

Published online: 10 January 2015

(C) Springer International Publishing AG 2015

This article is part of the Topical Collection on Specific Immunotherapy

Keywords Allergen - Allergic rhinitis - Immunotherapy - Local allergic rhinitis - Nasal allergen provocation test

\section{Opinion statement}

Allergen immunotherapy (AIT), the etiologic treatment of allergic rhinoconjunctivitis and allergic asthma, has been shown to be an effective and safe treatment in patients with allergic respiratory disease. The efficacy of AIT in reducing symptoms and medication requirements has been demonstrated not only during treatment but also after it is discontinued. AIT is the only treatment that has the ability to modify the natural course of allergic rhinoconjunctivitis and asthma. In recent years, a new entity-local allergic rhinitis (LAR) - has been reported, which is characterized by the presence of a local allergic response to inhalant allergens, with negative skin tests and no detection of specific IgE antibodies in the peripheral blood. Patients had a nasal-specific response after nasal allergen challenge and a nasal Th2 inflammatory response with specific IgE antibodies, and showed clinical improvement with the classical treatment for allergic rhinitis (antihistamines and nasal corticosteroids). Ongoing evidence indicates that these patients benefit from subcutaneous AIT and supports this indication for LAR.

Key points

1. LAR is a new phenotype of allergic rhinitis characterized by the presence of a localized allergic response in the nasal mucosa with negative skin test and no detection of serumspecific IgE antibodies in the peripheral blood.

2. It is an underdiagnosed/misdiagnosed respiratory disease that may affect patients from different countries and among different ethnic and age groups, showing a tendency 
toward worsening of the disease and a risk of developing asthma.

3. Patients with LAR commonly have persistent rhinitis, with moderate to severe nasal symptoms associated with conjunctivitis and/or asthma and impairment of their quality of life.

4. AIT is the etiological treatment of allergic respiratory disease and has the ability to modify its natural course. According to ARIA guidelines, it is indicated in moderate to severe persistent or intermittent allergic rhinitis and/or allergic asthma.

5. The efficacy and safety of subcutaneous allergen-specific immunotherapy for grass pollen and Dermatophagoides pteronyssinus has been demonstrated in adults with LAR.

\section{Introduction}

Allergic rhinitis (AR) is a global health problem, adversely affecting quality of life, and frequently associated with asthma, conjunctivitis, and rhinosinusitis [1]. Thus, the early diagnosis and effective management of AR can improve patients' quality of life and their clinical evolution.

In the past decade, a new phenotype of AR has been described, characterized by the presence of a localized allergic response in the nasal mucosa, with the absence of atopy assessed by conventional diagnostic tests such as skin-prick testing (SPT) and/or determination of

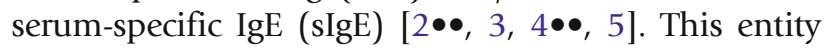
has been identified as local allergic rhinitis (LAR), or entopy $[4 \bullet \bullet, 6,7]$.

The immunological characteristics of LAR include in situ production of serum-specific IgE (sIgE) antibodies to inhalant allergens, a Th2 pattern of mucosal inflammatory response during natural exposure to aeroallergens, and a positive NAPT with release of inflammatory mediators $[2 \bullet \bullet, 3,4 \bullet \bullet, 8-11]$. LAR affects more than $45 \%$ of patients previously diagnosed with non-allergic

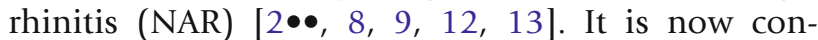
sidered a new phenotype of allergic rhinitis and is included in the AR group in the latest etiological classification of rhinitis $[3,4 \bullet \bullet, 14 \bullet \bullet]$.

The characterization of LAR has generated several important questions concerning its prevalence and natural evolution, the influence of environmental factors, and the role of immunotherapy. The evidence of a localized allergic response in patients with non-atopic rhinitis suggests that allergen-specific immunotherapy would be beneficial in these patients. This article review the latest work carried out on LAR, with a particular emphasis on the clinical and immunological effects of systemic AIT in LAR.

\section{Immunological characteristics of LAR}

There is sufficient evidence supporting the contention that LAR is a localized IgE-mediated allergic respiratory disease. The primary difference between classical systemic AR and LAR is that, in the former, the specific IgE is circulated in peripheral blood and can be detected in the sensitized mast cells of the skin (SPT), and is also a free antibody circulating in sera, whereas in LAR, it cannot be detected. The reasons, however, remain unknown.

Various studies have demonstrated that patients with LAR have a Th2 allergic inflammatory response, driven by nasal production of specific $\operatorname{IgE}[8,9,15,16]$ and nasal accumulation of eosinophils, mast cells, and CD3+/CD4+ T cells [8, 9, 15-17], as well as a positive response to nasal allergen provocation tests (NAPT) $[2 \bullet \bullet, 8,9,12,13]$. The immediate and late phases of the allergic response to NAPT have been measured in LAR patients through the monitoring of symptoms, acoustic rhinometry, and the release of inflammatory mediators [10, 11]. 


\section{Th2 allergic nasal inflammatory response}

In LAR, as in AR, the natural exposure to inhalant allergens is associated with a nasal Th2 IgE response. Increased levels of eosinophils, basophils, mast cells, CD3+ T cells, and CD4+ T cells have been detected by flow cytometry studies in nasal lavage $[8,9]$. T cells may contribute to the recruitment of eosinophils and to the production of the IgE antibodies in patients with LAR.

In the past, the hypothesis that an inflammatory mechanism might be involved in the pathophysiology of non-allergic rhinitis, however, has been controversial. Various histological studies have found a Th2 inflammatory pattern in patients with NAR compared to healthy individuals, with increased numbers of mast cells, eosinophils, $\operatorname{IgE}^{+} B$ cells [15], and T cells [16], although these findings have not been reported by others $[18,19]$. The reason for these apparently contradictory results may be the great heterogeneity of NAR, including patients with a different pathogenesis, predominantly inflammatory in some cases $[15,16]$, possibly including patients with LAR, and including neurogenic mechanisms in patients with IR $[18,19]$.

\section{Local production of sIgE and pro-inflammatory mediators}

Although the detection of nasal sIgE to D. pteronyssinus was reported by Huggins and Brostoff in 1975 [20] in a group of rhinitis patients with positive NAPT to D. pteronyssinus and negative SPT and serum sIgE, it was not until 2007 that nasal sIgE during natural exposure to an allergen was reported [8].

Over the years, researchers have demonstrated the presence of sIgE antibodies in nasal secretions of LAR patients during periods of natural exposure to aeroallergens $[8,9]$, after nasal challenge $[10,11,21 \bullet]$, and during periods of non-exposure to aeroallergens $[10,11]$. Cellular studies have found the expression of epsilon germline gene transcripts and mRNA for the epsilon heavy chain of IgE in B cells of the nasal mucosa [22]. In patients with negative skinprick tests, a Th 2 inflammatory pattern, with increased numbers of IgE+B cells, mast cells, and eosinophils, has been detected [15].

The presence of free light chains (FLCs) of IgE in the nasal mucosa and nasal secretions of patients with AR and NAR [23] suggests that these FLCs could play a role in IgE-mediated hypersensitive immune responses with the involvement of mast cells. Further investigation is needed to elucidate the presence of FLCs in patients with LAR and to establish whether FLCs have an adjuvant or independent role in patients with IgE-mediated allergic disorders.

In a recent study, Gómez et al. [24••] demonstrated that $50 \%$ of subjects with LAR to D. pteronyssinus had a positive and highly specific basophil activation test (BAT), supporting the concept that LAR is an IgE-mediated allergic disease and providing a new diagnostic tool. These results have been replicated

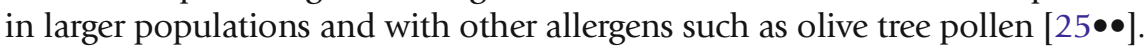
The peripheral basophil activation in LAR may be due to the spillover of locally synthesized sIgE into the circulation, with basophils the primary effector cells $[26 \bullet]$.

The kinetics of the immediate and late phases of the allergic response after NAPT has been studied in LAR subjects sensitised to grass pollen and D. pteronyssinus (DP) $[10,11]$. In these studies, the time course of clinical symptoms, reduction in nasal patency, and local production of sIgE and proinflammatory cytokines was evaluated $[10,11]$. Tryptase and eosinophil 
cationic protein (ECP) were chosen as specific markers of mast cell and eosinophil activation. Patients had an immediate or dual response to NAPTs, accompanied by release of tryptase, ECP, and sIgE in nasal secretions. In these patients, we did not observe isolated late responses [10,11].

The kinetic curve of tryptase showed a strong correlation with nasal symptoms of itching and sneezing, with different patterns for immediate versus dualresponders. The maximum level of tryptase was detected at 15 minutes, decreasing over time, and normalizing at six hours in immediate responders and at 24 hours after challenge in dual-responders [10,11].

Eosinophilic cationic protein stored in the granules of eosinophils and secreted after cell activation has been used as a specific marker for eosinophil activation in late responses to NAPT in subjects with allergic rhinitis, showing a significant increase from four to nine hours after the challenge [27, 28].

In patients with LAR, the release of ECP was detected 15 minutes after the NAPT, with a progressive increase, reaching the maximum nasal concentration at 24 hours after provocation in both immediate and dual-responders [10, 11]. The maximum levels of ECP and sIgE to grass pollen and D. pteronyssinus were recorded at 24 hours after the challenge, the final time point of the evaluation period. Whether nasal production of ECP and/or sIgE continues to increase after this time point must be explored in further studies.

The detection in some patients of basal levels of nasal sIgE during non-exposure periods, which progressively increased from 1 to 24 hours after allergen challenge, supports the existence of persistent local production of sIgE in nasal mucosa that rapidly increases after allergenspecific stimulation $[10,11]$.

In these kinetic studies, a significant correlation was observed between the increase in nasal levels of tryptase and ECP and the intensity of nasal symptoms. These correlations were more pronounced in LAR patients sensitized to grass pollen between tryptase and nasal itching and rhinorrhea, and between ECP and nasal obstruction [10].

The diagnosis of LAR is based largely on the demonstration of a positive

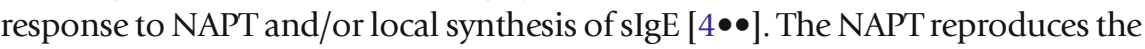
allergic reaction, allowing us to study the immediate and late allergic response demonstrating both the presence of local allergen-specific IgE and the triggering of the response by the allergen. The NAPT is considered the gold standard in the diagnosis of LAR, and has proven to be a highly sensitive, specific, and reproducible test, with a higher sensitivity than that associated with the detection of nasal-specific IgE antibodies [8, 9, 12]. This technique requires well trainedpersonnel, it is time-consuming, and it is not available in many centers. A multiple-allergen NAPT (NAPT-M) approach in a single session has been designed in order to shorten the procedure without impairing the specificity,

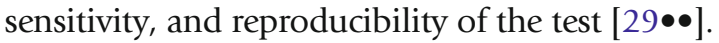

Several studies have shown that from $47 \%$ to $66 \%$ of patients previously diagnosed with idiopathic rhinitis or NAR were found to have LAR with positive NAPT responses measured objectively by acoustic rhinometry [8-11, 13], anterior rhinomanometry [12], and nasal secretion of sIgE and inflammatory mediators [8-11], and by subjective nasal and ocular symptoms $[8-13,14 \bullet \bullet$. 


\section{Natural history}

What is the natural history of LAR? Should it be considered a risk factor for asthma? These are important clinical question that were posed after LAR was first described. Whether this entity is a first step in the development of AR with systemic atopy or represents a distinct phenotype is currently under investigation.

In a retrospective study performed in 180 patients who were initially diagnosed with NAR between 2000 and 2004 and were reevaluated three to seven years later, the development of de novo sensitization was detected by means of skin-prick testing, serum-specific IgE, or both in $24 \%$ of the patients [30]. However this study did not differentiate between NAR and LAR patients.

In a follow-up study evaluating the natural history of LAR over a 10-year period in a cohort of 149 patients with LAR and 130 healthy controls, the results of the first five years of the study showed similarly low rates of conversion to systemic atopy in both patients and controls, suggesting that LAR is an entity well-differentiated from AR [31••]. Furthermore, after five years of follow-up, a worsening of rhinitis was detected, with a significant impairment in quality of life, an increase in the persistence and severity of nasal symptoms and in the use of emergency assistance, as well as new associations with conjunctivitis and asthma. After five years, con-

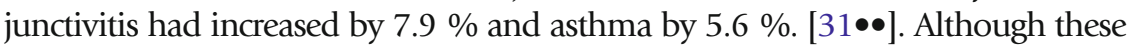
results must be validated after the completion of 10 years, the data show that LAR has a natural tendency toward worsening, with the appearance of new cases of asthma, although not evolving toward AR with systemic atopy.

\section{Phenotype and clinical relevance}

Although prevalence data in the general population are lacking, various publications have reported that LAR may affect more than $47 \%$ of patients previously diagnosed with NAR $[8-13,14 \bullet \bullet]$, and up to $25 \%$ of patients with

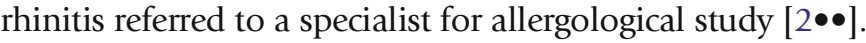

In recent years, several studies have shown that LAR is a common underdiagnosed/misdiagnosed respiratory disease that may affect patients

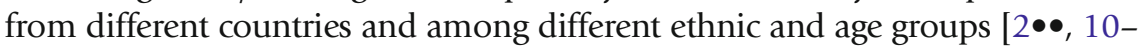
$12,21 \bullet, 32 \bullet, 33 \bullet]$.

Although data in childhood populations are limited, it has been suggested that LAR should be included in the differential diagnosis of rhinitis in children. In a recent study in children with perennial rhinitis and negative SPT, the authors found positive responses to NAPT with Alternaria in 30 of 36 children ( $83 \%$ ) [21•]. In another study including 110 adult patients with LAR, participants reported onset of the disease

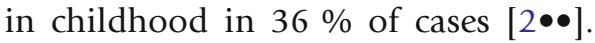

A recent study evaluated the clinical phenotype of patients with LAR in a comparative analysis of a large number of subjects with LAR, AR, and NAR

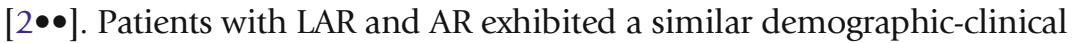
phenotype: a young woman, non-smoker, with severe persistent perennial rhinitis frequently associated with conjunctivitis and asthma, and with

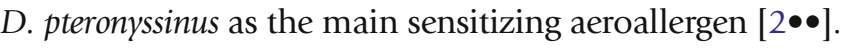




\section{Therapeutic options in local allergic rhinitis}

The management of allergic rhinitis includes patient education, allergen avoidance measures (when possible), pharmacotherapy, and AIT. The Allergic Rhinitis and its Impact on Asthma (ARIA) guidelines have proposed a new classification of allergic rhinitis and an initial treatment strategy based on the

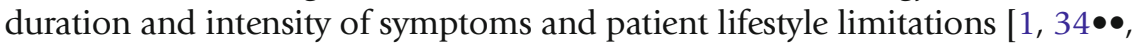
35].

Given the clinical-immunological similarities between patients with LAR and those with AR, it is reasonable to think that LAR patients would benefit from the same pharmacological treatment, including antihistamines, inhaled corticoids, and IgE antagonists, for nasal as well as bronchial symptoms.

In fact, the majority of patients with LAR are currently being treated with education, allergen avoidance measures, and pharmacological treatment with intranasal corticosteroids and oral antihistamines, according to the ARIA criteria $[8,9]$, and showing a similar response in terms of symptom relief and management of the disease [8, 9, 31••]. Allergen avoidance is not always suitable, however, and the efficacy of environmental avoidance measures is not well-established. In addition, intranasal corticosteroids and oral antihistamines cannot prevent progression of the disease.

AIT is the only treatment that has the ability to modify the natural course of the allergic disease in addition to providing a reduction in symptoms and the use of medication [36-38].

According to ARIA guidelines, allergen immunotherapy is indicated in children and adults with moderate to severe persistent or intermittent AR and/or allergic asthma [1, 34••, 35-38]. The long-term clinical effect of AIT and its potential role in preventing the development of asthma in patients with AR has been recognized in the ARIA $[1,34 \bullet \bullet, 35]$ and Global Initiative for Asthma (GINA) guidelines [39].

\section{Is there a role for systemic AIT in local allergic rhinitis?}

\section{The first steps - observational study}

The evidence of a localized allergic response in patients with non-atopic rhinitis suggests that allergen-specific immunotherapy would be beneficial for these patients. As discussed previously, patients with LAR often complain of persistent rhinitis with moderate-severe symptoms, frequently associated with conjuctivitis and asthma. Apart from the natural history, there is a worsening of the disease, with a tendency toward persistent and severe nasal symptoms, increased emergency assistance, and development of conjunctivitis and asthma $[31 \bullet \bullet]$. For these reasons, a proportion of these patients require continous pharmacological treatment with nasal corticosteroids and oral antihistamines for the relief of their symptoms $[8,9,31 \bullet \bullet]$.

In order to evaluate the potential role of AIT in local allergic rhinitis, an initial observational study was conducted by Rondón et al. in 2011 [40••]. In this study, 20 adult patients (aged 19-45 years) with moderate or severe LAR 
sensitized to grass pollen were evaluated. Ten patients were treated for six months with a pre-seasonal course of grass-specific subcutaneous immunotherapy (SCIT) and with rescue medication in the spring (active group), and 10 patients received rescue medication only (control group) [40••].

In the active group, the six-month course of pre-seasonal SCIT involved a six-week up-dose up-titration, followed by monthly subcutaneous administration of a standardized extract of a mixture of six grass pollens (Dactylis, Festuca, Lolium, Phleum, Poa, and Secale species), administered from September through March. Skin-prick tests, NAPT responses, and determination of levels of grass pollen-specific serum IgG and IgE to Lolium and Phleum species were performed at 1, 3, 6, and 12 months after beginning SCIT in order to evaluate the immune response. Seasonal symptoms and medication scores from April through June were recorded at baseline and after one year. The results of this study showed that grass SCIT was a safe and effective treatment that induced immunological changes and clinical improvement in patients with LAR. The patients who received SCIT showed significant improvement in nasal tolerance to the allergen compared with the control group, with higher threshold concentrations of grass pollen in NAPTs after 6 and 12 months of treatment, and a negative response to NAPT in 3 of 10 patients (30\%). Significant increases in Lolium- and Phleumspecific serum IgG antibodies were also detected. A significant reduction in symptoms and rescue medication, and an increase in the number of medication-free days, was observed in the active group compared with the control group. No systemic adverse reactions were observed in the active group. Although a placebo effect may have been present in this study, the important increase in nasal tolerance to NAPT and the increase in serum levels of Lolium and Phleum-specific IgG antibodies are objective parameters indicative of the beneficial effect of SCIT [40••].

The results of this study show that SCIT with grass pollen is a safe and effective treatment in subjects with LAR, with significant improvement in nasal tolerance to NAPTs and clinical response to natural exposure to the allergen $[40 \bullet \bullet]$.

\section{New questions - from observational to interventional studies}

In light of the interesting results of the observational study, research was conducted in the form of a randomized double-blind placebo-controlled (DBPC) clinical trial to investigate the efficacy and safety of subcutaneous AIT with D. pteronyssinus and grass pollen in patients with LAR $[41 \bullet \bullet]$.

The first DBPC parallel-group phase II clinical trial of subcutaneous AIT with D. pteronyssinus (SCIT-DP) conducted in LAR patients proved that SCIT-DP was an effective and well-tolerated treatment [41••] In this phase II clinical trial, 36 subjects with LAR were randomized to receive SCIT (Pangramin Plus D. pteronyssinus [ALK]) or placebo for 24 months. SCIT-DP produced significant clinical improvement, with a reduction in symptom and medication scores and an increase in the number of medication-free days, as well as an objective improvement in nasal tolerance to NAPT-DP, compared to placebo, with negative responses to 
NAPT-DP in $50 \%$ of the patients. This first DBPC phase-II study pro-

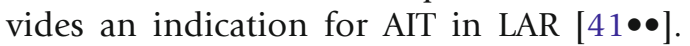

\section{Compliance with Ethics Guidelines}

\section{Conflict of Interest}

Carmen Rondón has had travel/accommodations expenses covered or reimbursed by EAACI.

Paloma Campo has had travel/accommodations expenses covered or reimbursed by EAACI.

Maria José Torres has had travel/accommodations expenses covered or reimbursed by EAACI.

Miguel Blanca has had travel/accommodations expenses covered or reimbursed by EAACI.

Natalia López-Blanca declares no conflict of interest.

\section{Human and Animal Rights and Informed Consent}

This article does not contain any studies with human or animal subjects performed by any of the authors.

\section{References and Recommended Reading}

Papers of particular interest, published recently, have been

highlighted as:

- Of importance

$\bullet$ Of major importance

1. Bousquet J, Van Cauwenberge P, Khaltaev N, Aria Workshop Group. World Health Organization. Allergic rhinitis and its impact on asthma. J Allergy Clin Immunol. 2001;108:S147-334.

2.• Rondón C, Campo P, Galindo L, et al. Prevalence and clinical relevance of local allergic rhinitis. Allergy. 2012;6:1282-8.

This study aimed to evaluate the prevalence, clinical characteristics, and severity of LAR in a rhinitis population compared with subjects with allergic rhinitis and non-allergic rhinitis.

3. Rondón C, Canto G, Blanca M. Local allergic rhinitis: a new entity, characterization and further studies. Curr Opin Allergy Clin Immunol. 2010;10(1):1-7.

4.• Rondón C, Campo P, Togias A, et al. Local allergic rhinitis: concept, pathophysiology, and management. J Allergy Clin Immunol. 2012;129(6):1460-7.

This work provides a presentation and discussion of the work that has been carried out on LAR in recent years, and controversies are highlighted. In addition, directions for future basic and clinical research are proposed.

5. Rondón C, Fernandez F, Canto G, Blanca M. Local allergic rhinitis: concept, clinical manifestations, and diagnostic approach. J Investig Allergol Clin Immunol. 2010;20:364-71.

6. Powe DG, Jones NS. Local mucosal immunoglobulin E production: does allergy exist in non-allergic rhinitis? Clin Exp Allergy. 2006;36(11):1367-72.

7. Powe DG, Bonnin AJ, Jones NS. 'Entopy': local allergy paradigm. Clin Exp Allergy. 2010;40(7):987-97.
8. Rondón C, Romero JJ, López S, et al. Local IgE production and positive nasal provocation test in patients with persistent nonallergic rhinitis. J Allergy Clin Immunol. 2007;119:899-905.

9. Rondón C, Doña I, López S, et al. Seasonal idiopathic rhinitis with local inflammatory response and specific IgE in absence of systemic response. Allergy. 2008;63:1352-8.

10. Rondón C, Fernández J, López S, et al. Nasal inflammatory mediators and specific-IgE production after nasal challenge with grass in local allergic rhinitis. J Allergy Clin Immunol. 2009;124:1005-11.

11. López S, Rondón C, Torres MJ, et al. Immediate and dual response to nasal challenge with

Dermatophagoides pteronyssinus in local allergic rhinitis. Clin Exp Allergy. 2010;40:1007-14.

12. Carney AS, Powe DG, Huskisson RS, Jones NS. Atypical nasal challenges in patients with idiopathic rhinitis: more evidence for the existence of allergy in the absence of atopy? Clin Exp Allergy. 2002;32:1436-40.

13. Wedbäck A, Enbom H, Eriksson NE, et al. Seasonal non-allergic rhinitis (SNAR)-a new disease entity? A clinical and immunological comparison between SNAR, seasonal allergic rhinitis and persistent nonallergic rhinitis. Rhinology. 2005;43:86-92.

14.• Papadopoulos NG, Bernstein J, Demoly P, et al. Phenotypes \& Endotypes of Rhinitis and their Impact on Management. European Academy of Allergy and Clinical Immunology /American Academy of Allergy, Asthma and Immunology/PRACTALL consensus 
report. Allergy (2014 submitted).

Consensus report that includes the definition and classification of different phenotypes and endotypes of rhinitis, and proposes diagnostic and therapeutic algorithms to be used in clinical practice

15. Powe DG, Huskisson RS, Carney AS, et al. Evidence for an inflammatory pathophysiology in idiopathic rhinitis. Clin Exp Allergy. 2001;31:864-72.

16. Powe DG, Huskisson RS, Carney AS, et al. Mucosal Tcell phenotypes in persistent atopic and nonatopic rhinitis show an association with mast cells. Allergy. 2004;59:204-12.

17. Powe DG, Jagger C, Kleinjan A, et al. "Entopy": localized mucosal allergic disease in the absence of systemic responses for atopy. Clin Exp Allergy. 2003;6:1374-9.

18. Blom HM, Godthelp T, Fokkens WJ, et al. Mast cells, eosinophils and IgE-positive cells in the nasal mucosa of patients with vasomotor rhinitis. An immunohistochemical study. Eur Arch Otorhinolaryngol. 1995;252 suppl 1:S33-9.

19. van Rijswijk JB, Blom HM, KleinJan A, et al. Inflammatory cells seem not to be involved in idiopathic rhinitis. Rhinology. 2003;41:25-30.

20. Huggins KG, Brostoff J. Local production of specific IgE antibodies in allergic- rhinitis patients with negative skin tests. Lancet. 1975;6:148-50.

21. Fuiano N, Fusilli S, Incorvaia C. A role for measurement of nasal IgE antibodies in diagnosis of Alternariainduced rhinitis in children. Allergol Immunopathol (Madr). 2012;6:71-4.

In this study, the authors detected the presence of LAR in children with positive response to NAPT with Alternaria and detection of nasal sIgE.

22. Durham SR, Gould HJ, Thienes CP, et al. Expression of epsilon germ-line gene transcripts and mRNA for the epsilon heavy chain of IgE in nasal B cells and the effects of topical corticosteroid. Eur J Immunol. 1997;27:2899-906.

23. Powe DG, Groot Kormelink T, Sisson M, et al. Evidence for the involvement of free light chain immunoglobulins in allergic and nonallergic rhinitis. J Allergy Clin Immunol. 2010;125:139-45.

e1-3.

24.• Gómez E, Campo P, Rondón C, et al. Role of the basophil activation test in the diagnosis of local allergic rhinitis. J Allergy Clin Immunol. 2013;132:975-6.e1-5. This work demonstrates a positive and highly specific basophil activation test (BAT) with D. Pteronyssinus, providing evidence that LAR is an IgE-mediated allergic disease and introducing a new diagnostic tool.

25.• Campo P, Rondon Segovia CM, Villalba M, Mayorga C, et al. Evaluation of in vivo and in vitro responses to $\mathrm{N}$ ole $\mathrm{e} 1$ in systemic and local allergic rhinitis. J Allergy Clin Immunology. 2013;131, Supp, AB237 (839).

This study reproduces the results of specific basophil activation in LAR with olive pollen allergen.

26. Dullaers M, De Bruyne R, Ramadani F, et al. The who, where, and when of IgE in allergic airway disease. J Allergy Clin Immunol. 2012;129(3):635-45.
In this study, the current knowledge regarding IgE in allergic respiratory disease is reviewed, with an emphasis the identification of IgE-secreting cells, their location, and the circumstances in which they are induced.

27. Graaf-in't Veld C, Garrelds IM, van Toorenenbergen AW, et al. Nasal responsiveness to allergen and histamine in patients with perennial rhinitis with and without a late phase response. Thorax. 1997;52:143-8.

28. Kramer MF, Jordan TR, Klemens C, et al. Factors contributing to nasal allergic late phase eosinophilia. Am J Otolaryngol. 2006;27:190-9.

29.• Rondón C, Campo P, Herrera R, et al. Nasal allergen provocation test with multiple aeroallergens detects polysensitization in local allergic rhinitis. J Allergy Clin Immunol. 2011;128:1192-7.

This important study showed that a new protocol of nasal allergen provocation testing with multiple allergens (NAPT-M) sequentially administrated in one session is a useful, specific, sensitive, reproducible, and less time-consuming diagnostic test for the screening of NAR and LAR.

30. Rondón C, Doña I, Torres MJ, Campo P, Blanca M. Evolution of patients with nonallergic rhinitis supports conversion to allergic rhinitis. J Allergy Clin Immunol. 2009; 123:1098-102.

31.• Rondón C, Campo P, Zambonino MA, et al. Follow-up study in local allergic rhinitis shows a consistent entity not evolving to systemic allergic rhinitis. J Allergy Clin Immunol. 2014;133(4):1026-31.

This first prospective follow-up study in LAR shows a similar rate of conversion to systemic atopy in LAR patients and controls, supporting the contention that LAR is a well-differentiated entity.

32.• Cheng KJ, Xu YY, Liu HY, Wang SQ. Serum eosinophil cationic protein level in Chinese subjects with nonallergic and local allergic rhinitis and its relation to the severity of disease. Am J Rhinol Allergy. 2013;27(1):812.

This study provides evidence of the existence of LAR in Chinese subjects. In this work, the authors demonstrated that LAR may affect patients in different countries and of ethnic groups.

33.• Oh J-W, Kim J-H, Cheong J-H. The differences Of TNFá, Rantes, Interleukin-5 levels in nasal polyps with allergic, local allergic, and non-allergic rhinitis. J Allergy Clin Immunol. 2014;133(2):AB128.

In this study, the presence of LAR in nasal polyposis was evaluated for the first time, detecting higher nasal levels of IL-5 and RANTES in allergic (AR and LAR) versus non-allergic polyp tissue homogenates. Another interesting finding was the detection of LAR in Korean subjects.

$34 . \bullet$ Brozek JL, Bousquet J, Baena-Cagnani CE, et al. Allergic rhinitis and its impact on asthma (ARIA) guidelines: 2010 Revision. J Allergy Clin Immunol. 2010;126:466-77.

Recent revision of the Allergic Rhinitis and its Impact on Asthma (ARIA) guidelines, first published in 2001. In this revision, the panel of authors has provided unified criteria for classification of patients and therapeutic options.

35. Bousquet J, Scünermann HJ, Samolinski B, et al. Allergic rhinitis and its impact on asthma (ARIA): 
achievements in 10 years and future needs. J Allergy Clin Immunol. 2012;130:1049-62.

36. Bousquet J, Lockey R, Malling HJ. Allergen immunotherapy: therapeutic vaccines for allergic diseases. A WHO position paper. J Allergy Clin Immunol. 1998;102:558-62.

37. Walker SM, Pajno GB, Lima MT, et al. Grass pollen immunotherapy for seasonal rhinitis and asthma: a randomized, controlled trial. J Allergy Clin Immunol. 2001;107:87-93.

38. Frew AJ, Powell RJ, Corrigan CJ, Durham SR, UK Immunotherapy Study Group. Efficacy and safety of specific immunotherapy with SQ allergen extract in treatment-resistant seasonal allergic rhinoconjunctivitis. J Allergy Clin Immunol. 2006;117:319-25.

39. Global Strategy for Asthma Management and Prevention. Global Initiative for Asthma (GINA) 2011. http:// www.ginasthma.org.
40.• Rondón C, Blanca-López N, Aranda A, et al. Local allergic rhinitis: allergen tolerance and immunologic changes after pre-seasonal immunotherapy with grass pollen. J Allergy Clin Immunol. 2011;127:1069-71.

This study is the first to examine the immunologic and clinical response to allergen-specific subcutaneous immunotherapy in patients with LAR sensitized to grass pollen. The results confirm the clinical improvement, immunologic modifying effect, and the safety of allergen-specific immunotherapy in these patients.

41.• Rondón C, Campo P, Blanca-López N, et al. Subcutaneous Allergen Immunotherapy in Patient with "Local Allergic Rhinitis" sensitized to Dermatophagoides pteronyssinus induces a significant clinical improvement. J Allergy Clin Immunol. 2015 (submitted). Randomized, double-blind, placebo-controlled, parallelgroup, phase II study investigating the efficacy and safety of subcutaneous AIT with Dermatophagoides pteronyssinus in patients with LAR 